\title{
„Performance Evaluation of Timed Events in Railways" in Österreich
}

\author{
Alexander Edthofer ${ }^{1 *}$, Martin Bicher ${ }^{2,3}$, Felix Breitenecker ${ }^{1}$ \\ ${ }^{1}$ Institute of Analysis and Scientific Computing, Vienna University of Technology, Wiedner Haupstraße 8-10, \\ 1040 Wien, Österreich; *alexander.edthofer@tuwien.ac.at \\ ${ }^{2} \mathrm{dwh}$ GmbH, Neustiftgasse 57-59, 1070 Wien, Österreich \\ ${ }^{3}$ Institute of Information Systems Engineering, Vienna University of Technology, Favoritenstraße 8-11, 1040 Wien, \\ Österreich
}

\begin{abstract}
Diese Arbeit befasst sich mit Methoden zur Verbesserung der Effizienz von Zugnetzen und wendet diese am Beispiel des österreichischen Güterzugverkehrs an. Ziel ist es, die Auslastung der Gleise zu analysieren und so den Fahrplan auf Realisierbarkeit zu untersuchen. Dies wird auf Güterzugfahrplandaten aus Österreich angewandt. Der theoretische Hintergrund ist die Max-Plus-Algebra, mit der ein Max-Plus Eigenwertproblem beschrieben werden kann. In dem Modell „PETER" wird dabei die zu untersuchende Matrix erstellt.
\end{abstract}

\section{Einleitung}

In den letzten Jahrzehnten hat der Zugverkehr im Personen- wie auch im Gütertransport auf der ganzen Welt enorm zugenommen. Während 1998 noch 179.465.000 Menschen in Österreich die Bahn nutzten, waren es 2018 bereits 309.534 .000 [7]. Güterzüge in unserem Land chauffierten 1998 noch 72.637.000 Tonnen, 2018 hingegen 105.271.000 Tonnen an Waren [7]. Da die Kapazität vieler Zugnetze bereits ausgeschöpft ist, müssen neue Möglichkeiten geschaffen werden, um die steigende Nachfrage weiterhin abdecken zu können.

Mit der Max-Plus Algebra lassen sich Modelle entwickeln, die insbesondere in Analyse und Optimierung von ereignisdiskreten Prozessen Anwendung finden, wie zum Beispiel bei Fahrplänen. In dieser Arbeit wird mit PETER ein solches Modell vorgestellt.

PETER ist die Abkürzung für ,Performance Evaluation of Timed Events in Railways", das heißt, es ist zur Effizienzanalyse von Zugfahrplänen entwickelt worden. Am Beispiel des dänischen Zugnetzes des Personenverkehrs ist dies in [3] angewandt worden.

Diese Arbeit beschäftigt sich mit dem Fahrplan des österreichischen Güterzugverkehrs. Aus den gegebenen
Daten der Abfahrt und Ankunft lässt sich eine quadratische Matrix erstellen, wobei die Anzahl der Zeilen und Spalten definiert wird durch die Anzahl der befahrenen Stationen. Durch Eigenwertanalyse dieser Matrix wird so der Fahrplan analysiert und auf Realisierbarkeit überprüft. Das Ziel der Arbeit ist nun, den Anteil des österreichischen Güterzugverkehrs am gesamten Eisenbahnverkehr einzuschätzen, um zu überprüfen, ob dieser Verspätungen verursacht.

\section{Max-Plus Algebra}

Die mathematische Grundlage für das folgende Modell bietet die Max-Plus Algebra. Auf der Algebra sind die Operationen $\oplus$ und $\otimes$ für $a, b \in \mathbb{R}_{\text {max }}$ folgendermaßen definiert, wobei max und + die herkömmlichen Operationen sind, welche aus den reellen Zahlen bekannt sind.

$$
\begin{aligned}
& a \oplus b:=\max \{a, b\} . \\
& a \otimes b:=a+b .
\end{aligned}
$$

Die Operationen $\oplus$ bzw. $\otimes$ besitzen die neutralen Elemente $\varepsilon:=-\infty$ bzw. 0. Weitere Eigenschaften der Max-Plus Algebra sind in [3, S. 13-20] nachzulesen.

Die Max-Plus Algebra lässt sich nun auch auf Vektoren und Matrizen in folgendem Sinn erweitern. Sei $\mathbb{R}_{\max }^{n \times m}$ die Menge aller $n \times m$ Matrizen mit Werten aus $\mathbb{R}_{\text {max }}$. Die Operationen können analog übernommen werden.

$$
[A \oplus B]_{i j}:=a_{i j} \oplus b_{i j}=\max \left\{a_{i j}, b_{i j}\right\} .
$$

Es wird also komponentenweise das Maximum der je- 
weiligen Einträge berechnet.

$$
[A \otimes B]_{i k}:=\bigoplus_{j=1}^{l} a_{i j} \otimes b_{j k}=\max _{j \in\{1, \ldots, l\}}\left\{a_{i j}+b_{j k}\right\} .
$$

Diese Operationen besitzen nun wiederum neutrale Elemente. So ist eine $n \times m$-Matrix, deren Werte alle gleich $\varepsilon$ sind, neutral bezüglich $\oplus$ und wird mit $\mathscr{E}(n, m)$ bezeichnet. Die Matrix $E(n, m)$, welche definiert ist als

$$
[E(n, m)]_{i j}:= \begin{cases}0 & i=j, \\ \varepsilon & \text { sonst }\end{cases}
$$

bildet das neutrale Element bezüglich $\otimes$. Für $n=m$ wird $E(n, n)$ auch Einheitsmatrix genannt. Für weitere Eigenschaften wird wiederum auf [3, S. 13-20] verwiesen.

\section{PETER - Performance Evaluation of Timed Events in Railways}

Ein geplanter Fahrplan der Eisenbahn kann als discrete event dynamic system (DEDS) mittels der Max-Plus Algebra modelliert werden. Hierzu werden irreduzible Modelle erster Ordnung der Form $x(k)=A \otimes x(k-1) \oplus$ $B \otimes u(k)$ betrachtet.

Um auch höhere Ordnungen, wie auch die nullte Ordnung, von Max-Plus linearen Systemen zu bearbeiten, wird im Folgenden eine „Polynom-MatrixRepräsentation"der Zustandsmatrix und der zugehörige zeitlich festgelegte Eventgraph vorgestellt. Dazu wird ein Shiftoperator $\gamma$ und seine Erweiterung auf Matrizen $A(\gamma)$ eingeführt. Die Theorie stützt sich großteils auf [2, S. 179 - 194], einige Informationen wurden auch aus [3, S. 36 - 45] entnommen. Dieser Operator führt uns schließlich auf eine Beschreibung ähnlich zu der MaxPlus Theorie erster Ordnung. Dieser Zugang wird nun verwendet, um periodische Eisenbahnfahrpläne als geplante Max-Plus lineare Systeme zu modellieren, und sie in weiterer Folge zu analysieren, um vor allem die Realisierbarkeit zu untersuchen.

\subsection{Aufbau des Modells}

Periodische Fahrpläne der Eisenbahn werden durch die in regelmäßigen Intervallen befahrenen Gleisstrecken bestimmt. Die Periodenlänge wird im Folgenden mit $T$ bezeichnet und beträgt üblicherweise 60 Minuten. In dem Modell (DEDS) beschreibt ein Event $i$ die Abfahrt eines Zuges der zugehörigen Linie $L_{i}$ aus der Station $S_{i}$. Weiters wird mit $x_{i}(k)$ die $k$-te Abfahrtszeit von $i$ bezeichnet, diese Zeit liegt also im Intervall $[k \cdot T,(k+1) \cdot T)$.

An $x_{i}(k)$ lassen sich nun einige Bedingungen stellen:

1. Fahrplanbedingung: Die Abfahrt darf nicht vor der geplanten Abfahrtszeit $d_{i}(k)$ erfolgen:

$$
x_{i}(k) \geq d_{i}(k) \text {. }
$$

Da der Fahrplan eine gewisse Periodenlänge hat, gilt $d_{i}(k)=d_{i}(0) \otimes T^{\otimes k}$.

2. Linienbedingung: Die Abfahrt eines Zuges hängt von den vorhergehenden Events auf der Linie ab:

$$
x_{i}(k) \geq a_{i j}+x_{j}\left(k-\mu_{i j}\right) .
$$

Dabei steht $a_{i j}$ für die Summe der Fahrzeit eines Zuges von der Station $S_{j}$ zur Station $S_{i}$ und die minimale Wartezeit in der Zielstation. Die periodische Verschiebung zwischen den Abfahrten $x_{i}$ und $x_{j}$ wird von $\mu_{i j} \in \mathbb{N}_{0}$ beschrieben. Sie wird mit der Aufrundungsfunktion berechnet:

$$
\mu_{i j}=\left\lceil\frac{a_{i j}+d_{j}^{0}-d_{i}^{0}}{T}\right\rceil .
$$

3. Synchronisationsbedingung: Weiterhin muss ein Zug oft auf andere warten. Berechnet wird dies analog zu (4), wobei $a_{i j}$ sich nun aus der Fahrzeit des ,Zulieferers“ und der Umstiegszeit in der Station $S_{i}$ zusammensetzt.

4. Infrastrukturbedingung: Schlussendlich muss ein Zug noch warten, falls das zu benutzende Gleis vor ihm blockiert ist. Wiederum gilt (4), wobei $a_{i j}$ nun für die Fahrtzeit nach Abfahrt steht, bevor die blockierte Stelle für den Zug $i$ freigegeben ist.

$\mathrm{Zu}$ beachten ist nun auch noch, dass $a_{i j}=a_{i j}\left(\mu_{i j}\right)$ von $\mu_{i j}$ abhängt, da die vorhergehende Abfahrt eventuell noch in einer alten Periode stattgefunden hat. Wenn nun $n$ die Anzahl der Abfahrtsevents ist und für jedes Paar $(j, i)$, an das keine Bedingung geknüpft ist $a_{i j}=\varepsilon$ gesetzt wird, lassen sich die Bedingungen als Gleichung

$$
\begin{aligned}
x_{i}(k) & =\bigoplus_{j=1}^{n}\left(a_{i j}\left(\mu_{i j}\right) \otimes x_{j}\left(k-\mu_{i j}\right)\right) \oplus d_{i}(k), \\
\forall i & =1, \ldots, n
\end{aligned}
$$


formulieren. Die Werte $a_{i j}$ lassen sich für gleiche periodische Verschiebungen zu Matrizen zusammenfassen: $A_{l}=\left(a_{i j}(l)\right)$ wiederum mit $\left[A_{l}\right]_{i j}=\varepsilon$, falls keine Bedingung in der jeweiligen periodischen Verschiebung existiert. Weiters lässt sich selbiges mit $x_{i}(k)$ und $d_{i}(k) \mathrm{zu}$ Vektoren machen. Die Ordnung des Systems wird im Folgenden mit $p \in \mathbb{N}$ bezeichnet. Damit kann nun (5) dargestellt werden:

$$
\begin{aligned}
x(k) & =\bigoplus_{l=0}^{p}\left(A_{l} \otimes x(k-l)\right) \oplus d(k), \\
d(k) & =d_{0} \otimes T^{\otimes k} .
\end{aligned}
$$

Die Gleichung (6) beschreibt nun die allgemeine $\mathrm{Zu}$ standraumdarstellung des geplanten Max-Plus linearen Systems mit periodischem Fahrplan, wobei $x(k)$ die wirkliche und $d(k)$ die geplante Abfahrtszeit ist.

Eine andere Formulierung von Max-Plus linearen Systemen höherer Ordnung kann mit einem ,backwardshift" $\gamma$ erhalten werden. Dieser ist definiert als

$$
\begin{aligned}
\gamma x(k)=x(k-1) \quad \text { bzw. } \quad \gamma^{l} x(k) & =x(k-l), \\
\gamma^{0} x(k) & =x(k) .
\end{aligned}
$$

Damit erhalten wir nun die alternative Beschreibung von (6)

$$
x(k)=A(\gamma) \otimes x(k) \oplus d(k)
$$

wobei $A(\gamma)=\bigoplus_{l=0}^{p} \gamma^{l} A_{l}$ eine polynomielle Matrix im Shift-Operator $\gamma$ ist.

\subsection{Eigenwertproblem}

Analog zur reellen Spektralanalyse lässt sich auch in der Max-Plus Algebra ein Eigenwertproblem definieren.

Für eine Matrix $A \in \mathbb{R}_{\text {max }}^{n \times n}$ heißt $\lambda \in \mathbb{R}_{\text {max }}$ Eigenwert und $v \in \mathbb{R}_{\text {max }} \backslash\{\varepsilon\}$ der zugehörige Eigenvektor, wenn

$$
A \otimes v=\lambda \otimes v
$$

Das Tupel $(\lambda, v)$ wird Eigenpaar und die Menge aller Eigenwerte Spektrum der Matrix $A$ genannt. Ein Algorithmus, welcher diesen berechnet, ist beispielsweise der Floyd-Warshall Algorithmus, welcher in Abschnitt 3 vorgestellt wird.
Weiters lässt sich das Eigenwertproblem auf polynomielle Max-Plus Matrizen verallgemeinern.

Definition 2.1 Sei $A(\gamma)=\bigoplus_{l=0}^{p} \gamma^{l} A_{l}$ eine polynomielle quadratische Max-Plus Matrix. Ein Skalar $\lambda \in$ $\mathbb{R}_{\text {max }} \backslash\{\varepsilon\}$ heißt Eigenwert und $v \in \mathbb{R}_{\text {max }}^{n} \backslash\{\varepsilon\}$ zugehöriger Eigenvektor $\Leftrightarrow$

$$
A\left(\lambda^{\otimes-1}\right) \otimes v=v .
$$

Eine homogenes Max-Plus lineares System $x(k)=A(\gamma) \otimes x(k)$ heißt autonom, wenn die polynomielle Zustandsmatrx $A(\gamma)$ keine Zeile besitzt, deren Werte alle gleich $\varepsilon$ sind, also $\forall 1 \leq i \leq n \exists 1 \leq j \leq n:[A(\gamma)]_{i j} \neq \varepsilon$. Eine quadratische polynomielle Matrix heißt irreduzibel, wenn sie zu einem autonomen Max-Plus linearen System gehört.

Um Max-Plus Spektralanalyse zu vertiefen, werden nun zeitbehaftete Petri-Netze (,timed petri nets“) vorgestellt. Dabei handelt es sich um einen Unterklasse von entscheidungsfreien Petri-Netzen mit nur einem Input beziehungsweise Output und ist ein DEDS. Das zeitbehaftete Petri-Netz ist ein Tupel $\mathscr{G}=(\mathscr{T}, \mathscr{P}, \mu, w)$, wobei $\mathscr{T}$ die Menge der Übergänge, also $|\mathscr{T}|=n, \mathscr{P}$ die Menge der Strecken mit $|\mathscr{P}|=m$ beschreibt. Die Variable $\mu \in \mathbb{N}_{0}^{m}$ ist eine Anfangsmarkierung, vergleichbar damit, wieviele Züge zu einem gewissen Startzeitpunkt verkehren bzw. verfügbar sind. Diese werden auch Token genannt. Mit $w \in\left(\mathbb{R}_{+} \cup \varepsilon\right)^{m}$ wird der Vektor der Fahrzeiten der einzelnen Strecken bezeichnet. Die Events, also die Übergänge, werden mit Balken, Strecken mit Kreisen dargestellt.

Ein Max-Plus lineares System ist die Zustandsraumrealisierung des zeitbehafteten Petri-Netzes. Die Verteilung der Token beschreiben den Zustand des Systems und die Bewegung der jener das dynamische Verhalten. Diese Bewegung geschieht nach folgender Regel: Ein Übergang ist aktiviert, wenn jede Strecke dorthin einen Token enthält und deren Fahrtzeit verstrichen ist. Danach ,feuert“ dieser Übergang, indem jede zuführende Strecke einen Token verliert, und jede wegführende einen erhält.

Eine polynomielle Matrix $A(\gamma)=\bigoplus_{l=0}^{p} \gamma^{l} A_{l}$ entspricht nun solch einem zeitbehafteten Petri Netz $\mathscr{G}$, mit $\mathscr{T}=$ $\{1, \ldots, n\}$, für jeden Wert $\left[A_{l}\right]_{i j} \neq \varepsilon$ gibt es eine Strecke $(j, i) \in \mathscr{P}$ deren Fahrtzeit $w_{i j}=\left[A_{l}\right]_{i j}$ ist. Die Anfangsmarkierung $\mu_{i j}$ beträgt $l$.

Weiters lässt sich nun der Begriff der Irreduzibilität auf die neue Darstellung anwenden: Die quadratische po- 
lynomielle Matrix $A(\gamma)$ heißt irreduzibel, wenn der zugehörige Graph $\mathscr{G}$ stark zusammenhängend ist. Solch eine Matrix hat nun einen einfachen verallgemeinerten Eigenwert, dem im Zusammenhang mit dem zeitbehafteten Petri-Netz eine besondere Rolle eingeräumt wird:

Satz 2.1 (Verallgemeinerter Eigenwert) Sei $A(\gamma)=$ $\bigoplus_{l=0} \gamma^{l} A_{l}$ eine irreduzible polynomielle Matrix mit einem gerichteten azyklischen Subgraphen. Dann hat $A(\gamma)$ einen einfachen verallgemeinerten Eigenwert $\lambda>$ $\varepsilon$ und einen Eigenvektor $v>\varepsilon$, sodass $A\left(\lambda^{\otimes-1}\right) \otimes v=v$, und $\lambda$ ist gleich der maximalen Zykluslänge des zugehörigen zeitbehafteten Petri-Netz $\mathscr{G}(A(\gamma))$,

$$
\eta=\max _{\xi \in C} \frac{w(\xi)}{\mu(\xi)},
$$

wobei $C$ die Menge aller grundlegenden Kreisläufe in $\mathscr{G}(A(\gamma)), w(\xi)$ die Kosten des Kreislaufs $\xi$ und $\mu(x i)$ die Zahl der Token in dem Kreislauf beschreibt.

Der Beweis ist in [2, S. 187] zu finden. Die Bedingung des azyklischen Subgraphen ist notwendig, da sonst Kreisläufe auftreten können, die keinen Token enthalten, und daher nicht mehr feuern können, womit möglicherweise kein Übergang, der von dort erreicht wird, jemals wieder aktiviert wird (festgefahren). Ein kritischer Kreislauf hat maximale Zykluslänge.

Ein Eigenvektor kann dann als AnfangsfahrplanVektor $d_{0}=v$ interpretiert werden, sodass das Max-plus lineare System eine Periodenlänge von $T=\lambda$ hat. Das Eigenwertproblem kann also auch folgendermaßen formuliert werden: Finde eine Wert $\lambda$, welcher die Zeiten der Wege $v_{i}$ von einem kritischen Knoten zu jedem Knoten $i$ minimiert (modulo $\lambda$ ).

\section{3 Österreichischer Güterzugverkehr}

Der Fahrplan des österreichischen Güterzugverkehrs ist sehr umfassend. Die Methode PETER wird nun auf diesen angewandt, welcher 1539803 Datensätze beinhaltet. Die Daten stehen wiederum im Rahmen des Projekts $A \& O$ mit den Österreichischen Bundesbahnen zur Verfügung. Der Fahrplan beinhaltet 40932 Züge, welche einerseits national, andererseits auch international verkehren.

Die Periode des untersuchten Fahrplans ist eine Woche, als Einheit sind Minuten gewählt. Damit ergibt sich für die Periode $T=10080$ min. Es lässt sich nun bereits erahnen, dass für diese Daten der Rechenaufwand um einiges länger ist, als bei der Analyse von Personenzügen, wie sie in [2] durchgeführt worden ist, bei der die Periode 60 Minuten ist, und außerdem pro Periodendurchgang viel weniger Züge zu untersuchen sind.

Für die Umsetzung der Methode PETER wird die Zugnummer, die Abfahrts- und Ankunftszeit, der Abfahrtsund Ankunftsort, die Anzahl der Zugzwischenstopps, wie auch etwaige Extrazeit aus dem Fahrplan benötigt. Letztere kommt bei Be- und Entladungen der Züge vor, oder aber auch wenn sie warten müssen, bis das Gleis vor ihnen frei wird. Um die Rechenzeit zu verkürzen, wird der Fahrplan soweit vereinfacht, dass nur mehr Start- und Endpunkt eines Zuges, sowie jene Informationen, wo eine Extrazeit stattfindet, gespeichert werden.

Von den gegebenen Daten werden nun zunächst die Anzahl der Züge bestimmt. Dann wird eine Matrix $W$ erstellt, für die ein Eintrag $w_{i j}$ das $i$-te wichtige Gleis des $j$-ten Zugs beschreibt. Diese Gleisstrecken werden in einen Vektor $G$ abgespeichert. Abschließend werden noch die Abfahrtszeiten und Ankunftszeit am Endbahnhof an den Wartestellen in die Matrix $Z$ geschrieben, deren Indizierung gleich der der Matrix $W$ ist.

Nun sind die Vorbereitungen getroffen, um die zu analysierende Matrix zu erstellen. Zunächst wird eine quadratische Matrix $A \in \mathbb{R}_{\max }^{n \times n}$, wobei $n$ gleich der Anzahl der wichtigen Gleise ist, erstellt. Anschließend werden gemäß den Bedingungen (3) und (4) die Einträge der Matrix auf das Maximum der zu wartenden bzw. fahrenden Zeit gesetzt. Im Vergleich zum theoretischen Modell von Abschnitt 2 liegt ein Problem der Modellumsetzung darin, dass nun mehrere Züge im Laufe der relativ langen Periode ein Gleisstück befahren und dies teilweise mit unterschiedlichen Geschwindigkeiten. Durch die Wahl der maximalen Zeit, was der geringsten Geschwindigkeit entspricht, wird das Modell so eher auf Realisierbarkeit des Fahrplans überprüft.

Der Eigenwert der Matrix wird nun mit dem FloydWarshall Algorithmus berechnet. Er beträgt $\lambda_{0}=2882$ min. Der Algorithmus läuft dabei folgendermaßen ab.

Wie in [5] erläutert, wird zunächst eine erste untere Schranke $\mu$ für den Eigenwert gewählt. Ist ein Wert $\operatorname{der}$ Diagonale von $A$ ungleich $\varepsilon$, so wird $\mu=\max _{i \in \underline{n}} a_{i i}$ 
gesetzt. Andernfalls ist $\mu=\min a_{i j}$ über alle $a_{i j} \neq \varepsilon$. In weiterer Folge wird überprüft, ob die Matrix $A-\mu J$, wobei $J \in \mathbb{R}^{n \times n}$ eine Matrix ist, deren Werte alle gleich 1 sind, Zyklen mit positiven Gewichten aufweist. Zyklen sind Pfade, deren Start- und Endpunkt derselbe ist. Dies wird untersucht, indem eine Folge von Matrizen $A_{k}$ konstruiert wird. Die erste Matrix ist dabei $A_{0}=A-\mu J$. Die weiteren Matrizen sind über

$$
\begin{aligned}
A_{k}(i, j) & :=\max \left\{A_{k-1}(i, j), A_{k-1}(i, k)+A_{k-1}(k, j)\right\}, \\
k & =1, \ldots, n,
\end{aligned}
$$

definiert. Ein Eintrag dieser Art kann als maximales Gewicht aller Pfade von $j$ nach $i$ der Länge $k+1$ gesehen werden. Innerhalb dieser Pfade wird nun auf $\mathrm{Zy}-$ klen geachtet, wobei jetzt nur mehr auf den Diagonalen der Matrizen $A_{k}$ geachtet werden muss. Wenn kein Wert der Diagonalen größer 0 ist, haben wir bereits den maximalen Eigenwert gefunden mit $\lambda_{0}=\mu$. Sollte es doch noch einen positiven Wert $\left(A_{k}\right)_{\tilde{i} i}$ an einer Diagonale geben, dann ist $\mu<\lambda_{0}$. Der zugehörige Zyklus, welcher von dem Knoten $\tilde{i}$ ausgeht, wird dann zurückverfolgt und $\mu$ durch das durchschnittliche Pfadgewicht $\frac{\text { Summe der Pfadgewichte }}{\text { Länge des Zyklus }}$ ersetzt. Die Prozedur wird daraufhin mit dem aktualisierten Wert von $\mu$ durchgeführt.

Um nun zugehörige Eigenvektoren zu finden, müssen zunächst theoretische Überlegungen gemacht werden. Ein kritischer Zyklus ist, wie in Abschnitt 2.2 bereits erwähnt, ein Zyklus mit maximaler Länge, dessen Länge also gleich $\lambda_{0}$ ist. Eine notwendige Bedingung für die Existenz eines Eigenvektors ist in folgendem Satz zu finden (siehe [5, S. 236]).

Satz 3.1 Wenn ein Eigenvektor $v$ des Problems $A \otimes v=$ $\lambda_{0} \otimes v$ mit $A \in \mathbb{R}^{n \times n}$ existiert, dann gibt es für alle $i \in \underline{n}$ Pfade im zur Matrix A gehörigen Graphen von einem Knoten eines kritischen Zyklus zu i.

Beweis. Das Eigenwertproblem $A \otimes v=\lambda_{0} \otimes v$ lässt sich auch kompenentenweise schreiben als

$$
\begin{aligned}
\max _{1 \leq j \leq n}\left(A_{i j}+v_{j}\right) & =\lambda_{0}+v_{i}, \quad 1 \leq i \leq n \\
\Longleftrightarrow \max _{1 \leq j \leq n}\left(A_{i j}+v_{j}-v_{i}\right) & =\lambda_{0}, \quad 1 \leq i \leq n .
\end{aligned}
$$

Von der Gleichung (8) ausgehend ist nun bekannt, dass für jeden Knoten $i$ mindestens ein $j$ existiert, sodass diese erfüllt ist. Dieser Knoten sei mit $\tilde{j_{1}}$ bezeichnet. Wenn dies nun weitergeführt wird, so gibt es für $\tilde{j}_{1}$ wiederum ein $\tilde{j_{2}}$, sodass (8) erfüllt ist. Iterativ entsteht so ein Pfad unendlicher Länge zu dem ursprünglichen
Knoten $i$. Da es aber nur $n$ Knoten insgesamt gibt, muss ein Zyklus enthalten sein, und da für jeden Pfad die Gleichung (8) gilt, ist dieser ein kritischer Zyklus.

Wenn nun die Kontraposition dieses Satzes betrachtet wird, so gilt nun, dass, wenn ein Knoten $\tilde{i}$ existiert, zu dem es keinen Pfad eines Knoten eines kritischen Zyklus gibt, dann gibt es keinen Eigenvektor. Dies ist in im gegebenen Beispiel des Güterzugmodells der Fall. Wenn nämlich Abbildung 1 betrachtet wird, fällt auf, dass darin drei Subsysteme vorhanden sind, was wiederum bedeutet, dass nicht alle befahrenen wichtigen Gleise miteinander verbunden sind. Der kritische Zyklus, welcher in dem linken Subsystem enthalten ist, erreicht zwar die meisten Stationen, jedoch eben nicht alle.

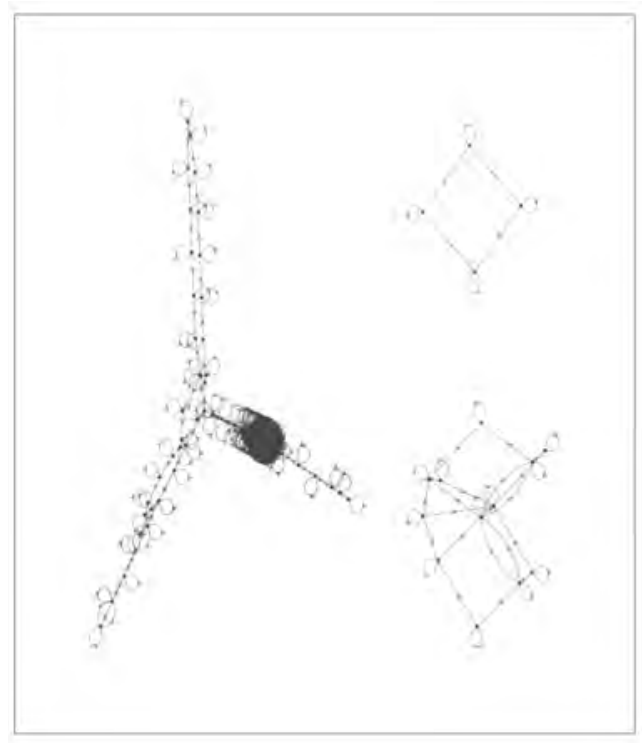

Abbildung 1: Der Güterzugfahrplan weist drei Subsysteme auf.

Was sagt uns der Eigenwert aber nun und in welchem Kontext muss er betrachtet werden? Zunächst einmal ist 
zu bemerken, dass eine Woche 10080 Minuten hat. Dadurch wirkt $\lambda_{0}=2882$ min zunächst sehr klein. Hierzu muss jedoch bedacht werden, dass nur Güterzüge betrachtet. Personenzüge, welche auf den gleichen Gleisen unterwegs sind und insbesondere bei eventuellen Wartezeiten Vorrang haben, werden nicht untersucht, da hierzu keine Daten zu Verfügung standen. Dies ist ein entscheidender Faktor, da laut [4] Personenzüge Österreichs Gleise mit insgesamt 114,9 Millionen Kilometer viel mehr belegen. Güterzüge haben im Gegensatz dazu „,nur“ 41,5 Millionen Kilometer zurückgelegt. Damit werden von den insgesamt gefahrenen 156,4 Millionen Kilometern 26,53\% von Güterzügen zurückgelegt. Wenn nun der Eigenwert betrachtet wird, so wird 28,59\% der Zeit von Güterzügen beansprucht.

Nun wirkt es zunächst so, als wäre das System instabil, da Güterzüge mehr Kilometer auf den Gleisen verbringen, als Personenzüge. Da sie durchschnittlich mit 90 bis 120 km/h zwar gleich schnell, wie Personennahverkehrszüge unterwegs sind, jedoch Personenfernverkehrszüge eine um einiges höhere Geschwindigkeit haben, ist die Zeit, welche Güterzüge auf den Gleisen verbringen, auch vergleichsweise länger (siehe [6, S.442449]). Weiters besitzen manche Gleisabschnitte mehrere Gleise nebeneinander, sodass mehrere Züge zur selben Zeit in die gleiche Richtung fahren können, ohne sich zu blockieren, was mehr Ressourcen schafft. Diese sind jedoch nicht in dem angegebenen Modell eingebunden. Damit relativiert sich der Unterschied zwischen den Prozentsätzen und das Ergebnis wirkt plausibel, der Fahrplan also realisierbar.

\section{Fazit}

In dieser Arbeit wurden die Max-Plus Algebra und darauf aufbauend ein Modell zur Zugfahrplananalyse und -optimierung vorgestellt. Insbesondere wird die Kapazitätsbelastung der Gleisnetze bewertet.

Das Modell Performance Evaluation of Timed Events in Railways (PETER) verkörpert einen graphentheoretischen Ansatz in Kombination mit einem MaxPlus Eigenwertproblem. Die Vorteile liegen nun darin, dass Nebenbedingungen wie Wartungen, Wenden, Koppeln, usw. kein extriges Modellieren benötigen, sondern die Züge auf dem momentanen Gleis länger verharren. Was bei diesem Modell im Anwendungsfall aufgefallen ist, ist, dass bei der Analyse des Güterzugfahrplans keine polynomielle Matrix, wie sie in Abschnitt 2 eingeführt wurde, benötigt wird, da durch die lange Peri- odendauer von einer Woche die Züge maximal mit jenen der nächsten Woche in Kontakt kommen, wodurch ein DEDS von erster bzw. nullter Ordnung ensteht. Das Modell lässt sich nun noch in ein paar Aspekten erweitern. So lässt sich der Fahrplan weitergehend auf Stabilität und Robustheit untersuchen. Stabilität bedeutet dabei, ob ein Fahrplan Verspätungen produziert, und Robustheit, ob diese wieder kompensiert werden können. Damit wird die Effizienz des Fahrplans getestet. Durch den Wegfall des Max-Plus-Eigenvektors konnte dies in dem beschriebenen Beispiel aber nicht angewandt werden. Für weitergehende Analyse würden auch noch Daten zur Geschwindigkeit von Güter- und Personenzügen benötigt werden, um den berechneten maximalen Eigenwert mit dem tatsächlichen zeitlichen Anteil von Güterzügen auf den Gleisen in Verbindung zu bringen.

\section{Referenzen}

[1] Borndörfer R., Klug T., Lamorgese L., Mannino C., Reuther M., Schlechte Th.: Handbook of Optimization in the Railway Industry. Bd. 268. Springer International Publishing; 2018.

[2] Goverde R.M.: Railway timetable stability analysis using max-plus system theory. In: Transportation Research Part B: Methodological. Elsevier; 2007; 2: p 179-201.

[3] Heidergott B., Olsder G.J., van der Woude J.: Max Plus at Work: Modeling and Analysis of Synchronized Systems: A Course on Max-Plus Algebra and Its Applications. Bd. 48. Princeton University Press; 2014.

[4] OEBB-Infrastruktur-AG:Zahlen, Daten, Fakten. https://infrastruktur.oebb.at/de/unternehmen/zahlendaten-fakten/zahlen-daten-fakten-oebbinfrastruktur.pdf. Letzter Zugriff am 7. Mai 2020.

[5] Olsder G.J., Roos K., Egmond R.-J.: An efficient algorithm for critical circuits and finite eigenvectors in the max-plus algebra. In: Linear Algebra and its Applications. Elsevier; 1999; 295: p 231-240.

[6] Röll F.V.v.: Enzyklopädie des Eisenbahnwesens : Vierter Band. Vero Verlag; 2019.

[7] United Nations Economic Commission for Europe: UNECE Statistical Database - Transport - Railway Traffic. https://w3.unece.org/PXWeb2015/pxweb/en/STAT/STAT_40TRTRANS_05-TRRAIL/. Letzter Zugriff am 25. Jänner 2020. 